\title{
Automated Acquisition of Proximal Femur Morphological Characteristics
}

\author{
Slobodan Tabakovic, Milan Zeljkovic, Zoran Milojevic \\ Faculty of technical sciences, University of Novi Sad, \\ Trg Dositeja Obradovica 6, 21000, Novi Sad, Serbia, \\ Email: tabak@uns.ac.rs
}

\begin{abstract}
The success of the hip arthroplasty surgery largely depends on the endoprosthesis adjustment to the patient's femur. This implies that the position of the femoral bone in relation to the pelvis is preserved and that the endoprosthesis position ensures its longevity. Dimensions and body shape of the hip joint endoprosthesis and its position after the surgery depend on a number of geometrical parameters of the patient's femur. One of the most suitable methods for determination of these parameters involves 3D reconstruction of femur, based on diagnostic images, and subsequent determination of the required geometric parameters.

In this paper, software for automated determination of geometric parameters of the femur is presented. Detailed software development procedure for the purpose of faster and more efficient design of the hip endoprosthesis that ensures patients' specific requirements is also offered.
\end{abstract}

Keywords: Automated acquisition, hip, morphological characteristics.

\section{INTRODUCTION}

$\mathrm{R}$ EPLACEMENT SURGERY of the natural hip joint with an artificial one (hip arthroplasty) is among the most commonly used procedures in orthopedic surgery. Conducted research indicates that over 800,000 procedures of this type are performed around the world every year [1]. Basic endoprosthesis design requirements are prosthesis longevity and as short as possible recovery period of the patient. In recent times, primary total hip joint replacement surgery (Total Hip Arthoplasty - THA) results in more than $90 \%$ of endoprostheses being successfully used even after 10 years of their functionality $[2,3]$.

The success of hip arthroplasty depends on the following factors: morphology of the affected limb, type and extent of disease, type of surgery and endoprosthesis structure. Prosthesis structure factors include level of adaptation of prosthesis elements to the patient, and its mechanical properties.

Planning of the hip replacement surgery for a particular patient involves defining the influencing factors which can be classified into two categories. The first group includes factors that are defined on the basis of the patient's medical analysis (type and extent of disease, patient's age and surgery method choices). Keeping these factors in mind, many different types of endoprostheses were developed in recent years that are characterized by ISO 7206-1 standard [4]. The second category includes dimensions that define the prosthesis element geometry. The most important in this group are dimensions and morphological features of the patient's pelvis and femur [5] and they are crucial for prosthesis design [6]. Dimensions of individual elements of the femur, as well as the shape and characteristics of its geometry are determined by femur reconstruction based on diagnostic images (CT, MRI) [7-9].

This paper presents software developed for the automated determination of geometric parameters of the femur. Software was developed on modular principles from two parts (subsystems); one subsystem was used for extraction of characteristic geometric parameters based on DICOM images; the other subsystem was utilized to determine femur parameters. Procedures that are used represent improved methods of diagnostic images processing, as well as the original design created from a detailed analysis of the hip joint endoprosthesis design process according to the patient's requirements.

\section{SUBJECT \& METHODS}

\subsection{Reconstruction of femur geometry.}

The main source of input information in the design of hip endoprosthesis, considering bones morphology, are tomographic images of the pelvic region $[10,11]$ resulting from CT (Computer Tomography) and MRI (Magnetic Resonance Imaging) methods. Reconstruction of bones' external and internal geometry creates preconditions for: sizing of prosthesis elements $[1,11]$, optimization of elements of its geometry [10-12] and verification of the designed prosthesis by using various engineering analysis tools (e.g., finite element analysis [13, 14]).

In modern medical diagnostics, archiving of images is performed by using DICOM (Digital Imaging and Communication in Medicine) format records, covered by ISO 12052 [15]. Processes of skeleton element reconstruction based on DICOM files generally consist of three stages:

- Image pre-processing,

- Femur contours extraction,

- Definition of bones' geometric parameters [8].

Image pre-processing involves import and processing of metadata (containing information about the object and recording parameters) and upload of a series of planar images from the DICOM file. In addition, correction of contrast of individual images is performed [16] in order to emphasize the elements of the skeletal system. For this 
purpose, distribution of pixel contrast method is used (contrast for images can be from $I_{\min }$ to $I_{\max }$ ) for the entire range of depths (from $I_{d 0}=0$ to $I_{d m a x}=2^{\text {depth }}$ ). This results in a correction coefficient, $\mathrm{k}$ (in (1)), which is used to multiply each pixel of the image.

$$
k=\frac{2^{\text {depth }}}{l_{\text {max }}-l_{\text {min }}}
$$

For determination of the image depth range and limits of generated contrasts, information gathered from DICOM images which were saved in the metadata file is used.

Fig.1. shows the procedure for correction of images based on recording parameters and the desired contrast.

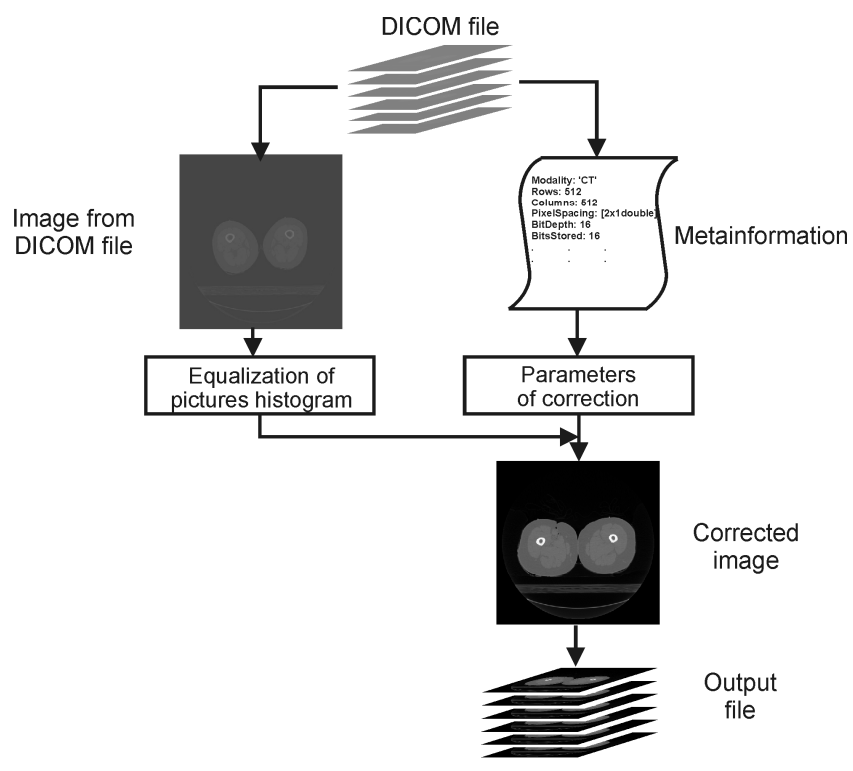

Fig.1. Image correction.

One of the main goals of this computer program is to determine geometric parameters that are required for the prosthesis design, which are most easily obtained by mathematical analysis of individual image sections. Because of this, femur contour extraction in this specific case is based on the 2D segmentation of the bone tissue [17], by filter application in order to detect edges, with subsequent isolation of the area of interest (i.e., bone contours) and noise correction that arise as a result of this procedure. In the segmentation procedure, the LoG (Laplacian of Gaussian) algorithm is used, which is based on edge definition of the object, derived from the rate of change of pixel contrast across the image. This algorithm is one of the methods for determination of objects' edges, based on the Laplace algorithm that defines the zero value of second derivative of pixel's contrast intensity function $\mathrm{I}(\mathrm{x}, \mathrm{y})$ on the image ( $\mathrm{x}$ and $\mathrm{y}$ determine position of pixels), and is defined by (2).

$$
L(x, y)=\frac{\partial^{2} I(x, y)}{\partial x^{2}}+\frac{\partial^{2} I(x, y)}{\partial y^{2}}
$$

Since the intensity contrast of raster images is represented by discrete values for every pixel, image matrix is multiplied by the corresponding convolution matrix. In order to reduce the noise that this kind of image processing generates, prior to Laplace algorithm application it is necessary to perform reduction of transitions by using the Gaussian filter described by equation (3).

$$
G(x, y)=\frac{2}{2 \pi \sigma^{2}} e^{-\frac{x^{2}+y^{2}}{2 \sigma^{2}}}
$$

The result of image processing is a series of contour lines that describe objects in any particular image. After segmentation, the object's boundaries are expanded and image contours closed by connecting the adjacent pixels. In the final phase, extraction of areas of interest is performed (in order to select external and internal geometry of the bone) as well as the removal of any existing noise.

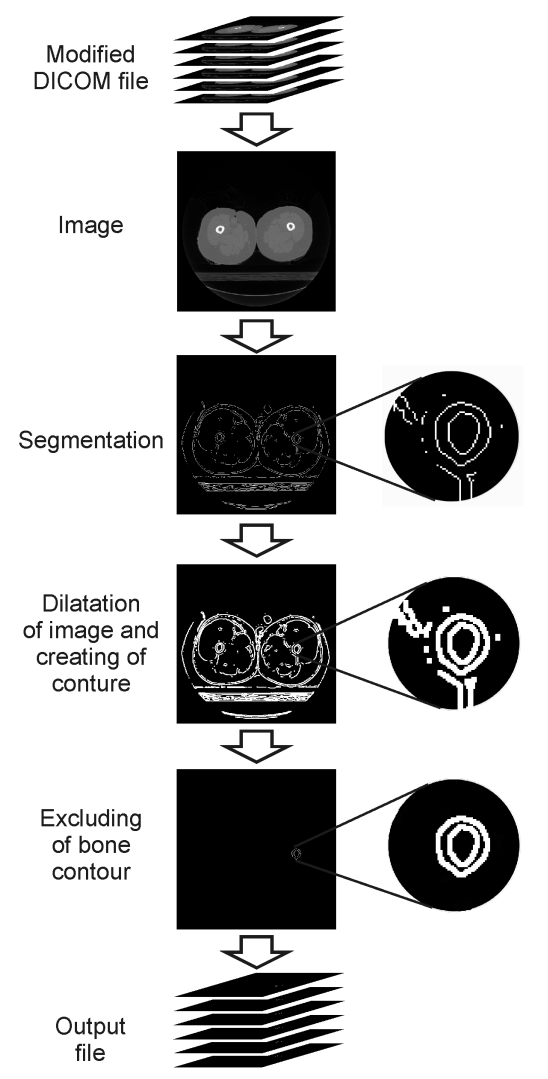

Fig.2. Image segmentation.

As a result of this image processing phase, a series of images that include contours of the selected bone are obtained.

Applicability of bone reconstruction procedure primarily depends on recording method and parameters, noise quantity, characteristics of the recording device and reconstruction method. Depending on the method and recording parameters, the accuracy of bone reconstruction may be significantly under $1 \mathrm{~mm}[18,19]$, which satisfies practical needs in any prosthesis design. 


\subsection{Determination of geometric parameters of the femur.}

The shape and dimensions of the hip endoprosthesis are defined on the basis of the number of parameters of external and internal geometry of the femur [11]. Regarding the influence on the prosthesis shape, these parameters can be categorized as global and local (Fig.3.).

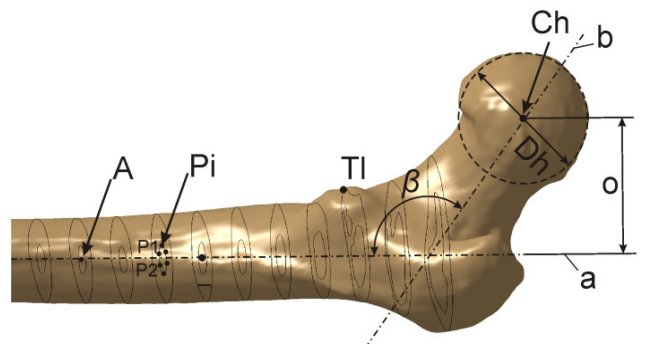

Fig.3. Femur morphology parameters.

Global parameters (or reference parameters) present the footprint for defining dimensions of the endoprosthesis and its optimal position in the femur. This group of parameters includes:

- Position of the medullary canal narrowing (A), isthmus, which determines position of the prosthesis' coordinate system, its length etc.,

- Anatomical axis of the femur (axis a),

- Position of the femoral head center (point $C_{h}$ ),

- Femoral head diameter $\left(\mathrm{D}_{\mathrm{h}}\right)$,

- Femoral neck axis (axis b)

- Distance between the axis and the femoral head center (offset) (o)

\section{- Angle of the femoral neck axis $(\beta)$}

The above geometric parameters are used as sources for preliminary definition of individual segments of the endoprosthesis body, in form of coordinates (position points), discrete algebraic values (length, diameter and angle), and mathematical rules (axes).

Local parameters are formed on the basis of femoral internal geometry and they portray the shape and dimensions of the medullary canal in typical cross-sections. These parameters present positions of corresponding points in characteristic femoral cross-sections (position $\mathrm{P}_{\mathrm{i}}$ in Fig.3.).

Proper processing of diagnostic images and reconstruction of femoral geometry in the form of spatial array (Cloud) of geometric elements (usually points) makes it possible to specify certain parameters (Fig.3.).

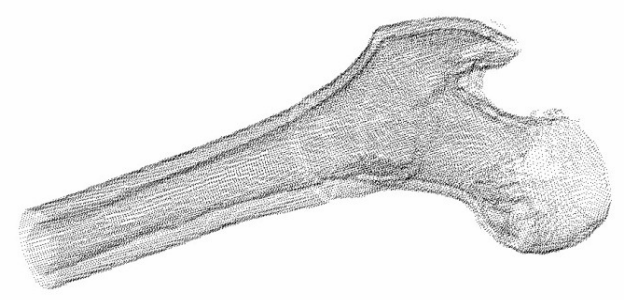

Fig.4. Cloud of points after femur reconstruction.
Local parameters are used to define the cross-sectional shape of endoprosthesis. These parameters in combination with the characteristic points of the external geometry allow the formation of a CAD model of the femur, which is used for verification of endoprosthesis shape and preoperative planning.

\subsubsection{Procedure for determination of femoral parameters.}

As a part of this research, various methods of numerical mathematics are applied to determine geometric parameters of the femur from a cloud of points.

Medullary canal narrowing is determined by calculation of minimum diameter of the circle inscribed in the medullary canal profile, for all image layers. Since the medullary canal cross-section is described by planar points, the center position and parameters of an inscribed circle are obtained by the least squares method. Optimal circle parameters are determined by the minimum sum of squares of the parameters applied to the planar circle equation (4) for $m$ points.

$$
\sum_{i=1}^{m} e^{2}=\sum_{i=1}^{m}\left\{\sqrt{\left(x_{i}-x_{c}\right)^{2}+\left(y_{i}-y_{c}\right)^{2}}-r\right\}^{2}
$$

The minimum value of this sum is obtained by determination of values $r, x_{\mathrm{c}}$ and $y_{\mathrm{c}}$, obtained by setting the partial first derivative of equation (4) for all variables [20] to zero. From the group of resulting circles, medullary canal narrowing is considered to be a circle with the smallest radius.

Femoral anatomical axis is obtained by using the same mathematical methods on the line equation in space, which defines the linear validity based on a set of $n$ points which are used to determine the position of the center of inscribed circles in the medullary canal profile (obtained by previously described procedure) (5).

$$
\sum_{i=1}^{n} e^{2}=\sum_{i=1}^{n}\left\{A x_{i}+B y_{i}+C-z_{i}\right\}^{2}
$$

Due to the femoral internal geometry, characteristics and change of the femoral structure in the proximal part, images of the femoral section from the narrowing to the position 20 $\mathrm{mm}$ below the lesser trochanter are used for defining anatomical axes [10].

Center position and femoral head diameter are determined by the least squares method applied to the sphere equation, for a set of $u$ points used to describe the femoral head or a part of it (6).

$\sum_{i=1}^{u} e^{2}=\sum_{i=1}^{u}\left\{\sqrt{\left(x_{i}-x_{c}\right)^{2}+\left(y_{i}-y_{c}\right)^{2}+\left(z_{i}-z_{c}\right)^{2}}-r\right\}^{2}$

In this case, the unknown $r, x_{\mathrm{c}}, y_{\mathrm{c}}$ and $z_{\mathrm{c}}$ values are calculated from a system of four equations that are obtained by setting the first partial derivative of equation (6) for all variables to zero.

In practice, some parts of the femoral head are often reconstructed with an error that occurs due to the small clearance between the femoral head and the pelvis, so the least squares method gives the best results. 
Femoral neck axis is determined by the cone that approximates the set of points on the femoral neck. For determination of femoral neck angle, femoral axis vector is used (Fig.5.).

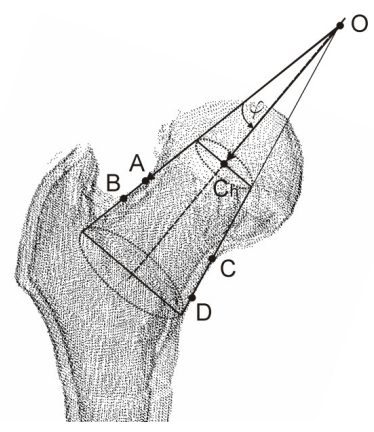

Fig.5. Determination of the femoral neck axis.

Based on any selected four points (A, B, C and D) on the surface of the femoral neck, and coordinates of the femoral head center $\mathrm{Ch}$ (which is part of the femoral neck axis), top point of the cone $(\mathrm{O})$ can be defined by using a system of four non-linear equations. Equation (7) describes the equation for point $\mathrm{A}$.

$$
\begin{gathered}
\text { const }=|\overrightarrow{O A}| * \cos \varphi=\frac{\overrightarrow{O A} * \overrightarrow{O C_{h}}}{\left|\overrightarrow{O C_{h}}\right|}= \\
=\frac{\left(x_{A}-x_{O}\right)\left(x_{C_{h}}-x_{O}\right)+\left(y_{A}-y_{O}\right)\left(y_{C_{h}}-y_{O}\right)+\left(z_{A}-z_{O}\right)\left(z_{C_{h}}-z_{O}\right)}{\sqrt{\left(x_{C_{h}}-x_{O}\right)^{2}+\left(y_{C_{h}}-y_{O}\right)^{2}+\left(z_{C_{h}}-z_{O}\right)^{2}}}
\end{gathered}
$$

Femoral axis vector is determined by coordinates of $\mathrm{C}_{h}$ and $\mathrm{O}$ points $(8)$.

$$
\overrightarrow{a_{f n}}=k \cdot \overrightarrow{O C_{h}}=k\left(\begin{array}{l}
x_{c h}-x_{o} \\
y_{c h}-y_{o} \\
z_{c h}-z_{o}
\end{array}\right)
$$

The distance between the axis and the femoral head center (offset) is determined by a well-known method for determination of minimum distance $(\mathrm{d})$ between the point and the vector (of the femoral axis) (Fig.6.).

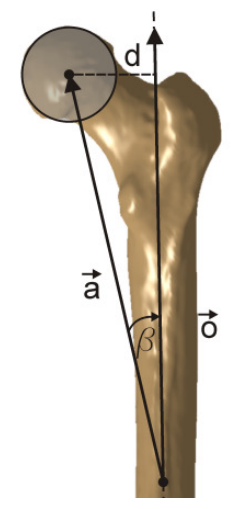

Fig.6. Determination of the distance between the femoral head center and femoral axis.
The method is based on the cross product of femoral axis vector $(\vec{O})$ and the vector that extends between the medullary canal narrowing point and the position of the femoral head center ( $\vec{a}$, known as the real femoral neck axis [21]), and can be described by (9).

$$
d=|\vec{a}| \sin \beta=\frac{|\vec{a} \times \vec{o}|}{|\vec{o}|}
$$

\section{DEVELOPED SOFTWARE}

\subsection{The overall structure of developed software.}

To achieve easier and more successful acquisition of geometric information that is necessary for the endoprosthesis design, specialized software that allows partial automation of femoral parameter determination is developed. The software is developed in MATLAB 2010, using specially developed functions in order to automate the procedure of femoral reconstruction and determine morphological parameters. To facilitate any further improvements, software structure is developed by using modular principles and it consists of two subsystems and multiple modules. Each developed module has a purpose to realize individual tasks in the image processing procedure and calculate femoral parameters.

In a global sense, the software was implemented by using two subsystems: subsystem for import and diagnostic image processing and subsystem for calculation of geometric parameters of the femur. Fig.7. is showing a global model of the developed software.

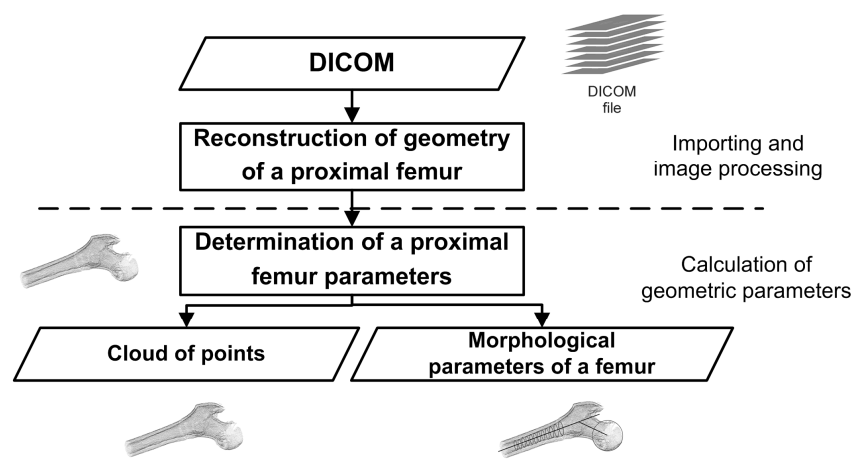

Fig.7. Model of the implemented software.

To additionally improve and partially test the results of individual modules, software creates output data files from each module. Output from subsystem that is used to reconstruct femoral geometry is a $3 \mathrm{D}$ matrix which describes the external and internal geometry of the femur. The output files from the other subsystem are point clouds matrix (adapted to communicate with commercial CAD systems) in ASCII format, and femoral morphological parameter matrix (which are specific to their positions and mathematical laws that describe them). 


\subsection{Reconstruction of femoral geometry.}

Input for the developed software is the subsystem for diagnostic image processing, composed of modules developed for the purpose of importing and processing of DICOM images. This process is executed in three stages that are implemented in different modules (Fig.8.). It is therefore possible to easily verify and evaluate the performance of individual image processing stages.

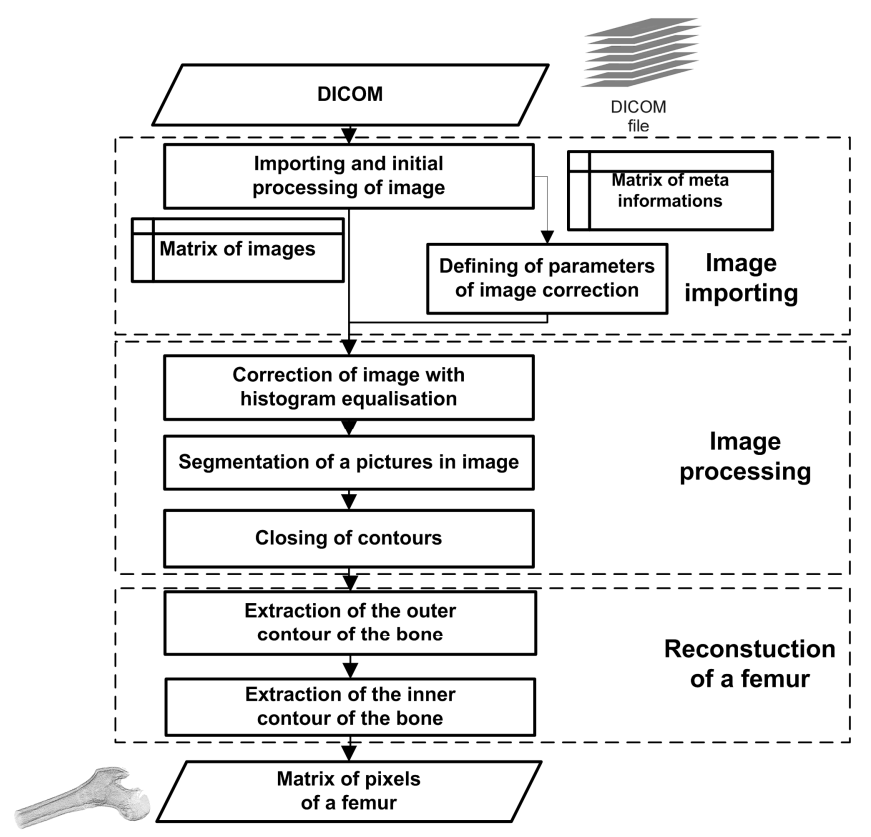

Fig.8. Processing and image reconstruction.

Image importing phase is implemented by converting each image into an appropriate $3 \mathrm{D}$ matrix containing the brightness intensity of each image pixel in order to form the metadata matrix. Metadata matrix contains information about: patient, recording parameters and characteristics of each image.

As a result of segmentation in the image processing stage, an array of binary images is obtained, where pixels contain the value of 1 in places that are defined by segmentation as object edges, and value of 0 for all other pixels. The whole record is described by the $3 \mathrm{D}$ matrix, whose dimensions correspond to the resolution of each image (usually $512 \times 512$ ) and to the number frames in the recording (i.e., recording layers). In addition, further processing of binary images is performed in this stage by closing the object lines, which is carried out by forming an object gradient mask. Finally, in the last stage of image processing, a desired contour is selected and objects that are caused by recording noise near outer and inner contours are removed. These objects are most frequently found in the proximal part of the femur, in the region of the medullary canal end as well as in the area of the femoral head. The phase of unwanted object removal is done manually by defining a polygonal mask that frames the object of interest.

As a result of image processing, a 3D matrix is obtained which contains bone contours description in binary form. This matrix is then forwarded for further processing.

\subsection{Determination of femoral parameters.}

Second part of the software is a subsystem that has a dual role: the creation of femur models in such a format that is suitable for processing in CAD software systems and determination of prosthesis parameters. Femur models in suitable format are realized by forming point clouds in standard ASCII format. This creates a base for prosthesis model analysis as a part of the femur to which it is fitted by application of Finite Element Method software. More important role of the subsystem, considering the design process, is calculation of geometric parameters of femoral external and internal geometry, which are very important for the endoprosthesis design process. This segment is also formed from a number of modules that are used to determine:

- Position of the lesser trochanter

- Positions of centers and radii of the maximum inscribed circle in the intersection of femoral medullary canal

- Positions of medullary canal narrowing (minimum inscribed circle) and femoral axis equation of femur internal geometry

- Positions of center and radius of the femoral head

- Femoral neck axis

- Femoral neck angle

- Distance from the femoral head center to the femoral axis (offset)

Fig.9. shows the structure of the module for femur size determination.

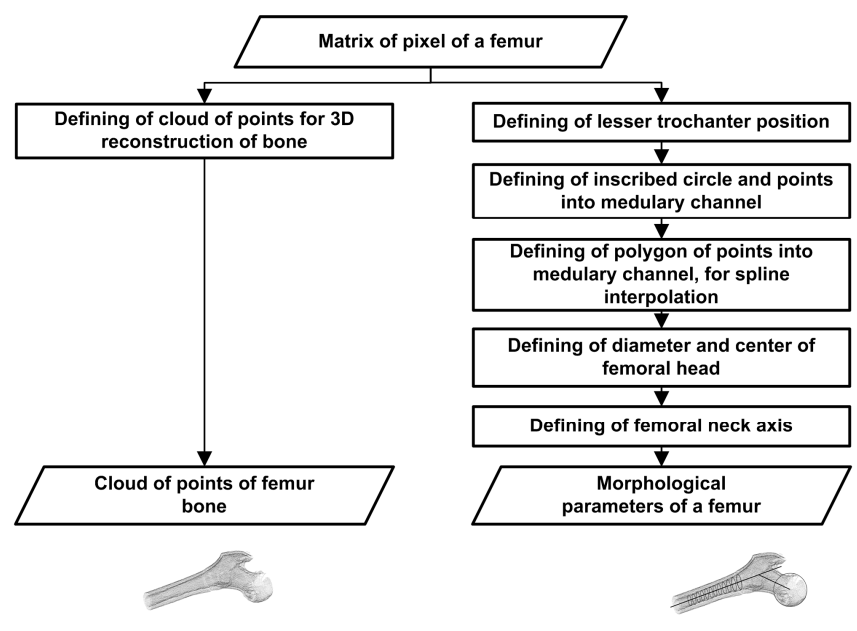

Fig.9. Determination of femur parameters.

Because of the complexity of the automated process for defining position of the lesser trochanter and selection of femoral head points, their determination is carried out with the assistance of software users, while the other parameters are determined automatically.

\section{ACQUISITION RESULTS}

Verification of applied methods and developed software involves processing a number of diagnostic recordings and analysis of attained data. 
In order to verify the developed software, 12 femurs were analyzed by processing DICOM images of patients of the Clinical Center of Vojvodina, Novi Sad, Serbia. The recordings include 9 partial and 3 complete DICOM files. Partial images were used for verification of femoral reconstruction (to the extent of image coverage) and determination of some parameters. Complete recordings were used to define all other parameters. Fig.10. depicts the method of software verification.

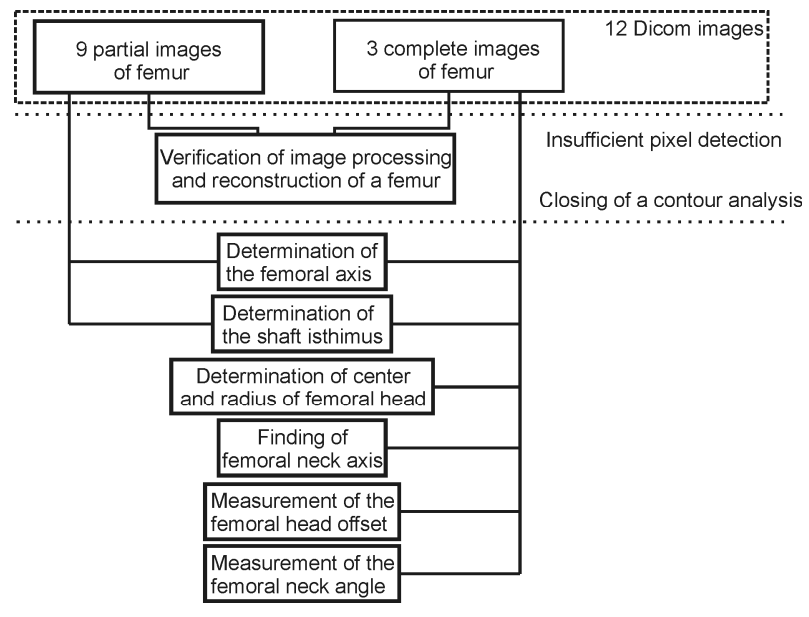

Fig.10. Software verification.

Table 1. Results of complete images analysis.

\begin{tabular}{|c|c|c|}
\hline Image & $\begin{array}{l}\text { Number of excess } \\
\text { pixels / total } \\
\text { number of pixels }\end{array}$ & $\begin{array}{l}\text { Number of images with } \\
\text { incomplete contour } \\
\text { closures / total number } \\
\text { of images }\end{array}$ \\
\hline CI-1 & $25 / 42549 \quad(0.06 \%)$ & $12 / 272 \quad(4.4 \%)$ \\
\hline CI-2 & $38 / 38810 \quad(0.10 \%)$ & $10 / 201 \quad(4.97 \%)$ \\
\hline CI-3 & $30 / 40342 \quad(0.07 \%)$ & $9 / 250 \quad(3.6 \%)$ \\
\hline
\end{tabular}

First part of verification involves functional analysis of subsystems for diagnostic image processing. It includes result analysis regarding any appearance of excess pixels in individual, processed images and incomplete contour closures of bone section. Occurrence of these irregularities in some parts of reconstructed femur may adversely affect determination of geometric parameters, e.g., calculation of the radius of the circle inscribed in the medullary canal. Analysis of reconstructed femurs has shown that the partial images, PI (which include the femoral body), had no irregularities. On the other hand, analysis of complete images, CI, showed that irregularities occur in the proximal part (as a result of appearance of several elements of the femur on the image, i.e., parts of greater trochanter and the femoral head), while the femoral body contained no irregularities. Table 1. presents results of complete image analysis.

Second part of verification includes defining of processed femoral morphological parameters. As Fig.10. shows, the partial images are used to define femoral axis and medullary canal narrowing, whilst complete images are used for determination of parameters included in the software. Table 2. presents parameters specified on femoral body, in the coordinate system defined by the image.

Table 2. Medullary canal parameters of analyzed femurs.

\begin{tabular}{|c|c|c|}
\hline Image & $\begin{array}{l}\text { Position }(x, y, z) \text { and } \\
\text { narrowing diameter } \\
{[\mathrm{mm}]}\end{array}$ & Axis equation \\
\hline CI-1 & $351,235,23 / 6.708$ & $\begin{array}{c}x=268-0.0145 t \\
y=385-0.0145 t \\
z=30+t\end{array}$ \\
\hline CI-2 & $385,264,3 / 7.071$ & $\begin{array}{c}x=239+0,2462 t \\
y=367+0,1231 t \\
z=110+t\end{array}$ \\
\hline CI-3 & $320,244,10 / 7.110$ & $\begin{array}{c}\mathrm{x}=255-0.143 \mathrm{t} \\
\mathrm{y}=373.4+0.088 \mathrm{t} \\
\mathrm{z}=78.8+\mathrm{t}\end{array}$ \\
\hline PI-1 & $442,281,15 / 7.701$ & $\begin{array}{c}\mathrm{x}=298-1.857 \mathrm{t} \\
\mathrm{y}=442-2.143 \mathrm{t} \\
\mathrm{z}=19.5+\mathrm{t}\end{array}$ \\
\hline PI-2 & $382,253,10 / 9.487$ & $\begin{array}{c}\mathrm{x}=253+0.846 \mathrm{t} \\
\mathrm{y}=382+0.923 \mathrm{t} \\
\mathrm{z}=10+\mathrm{t}\end{array}$ \\
\hline PI-3 & $416,260,3 / 8.861$ & $\begin{array}{c}x=256+0.142 t \\
y=385+0.184 t \\
z=2+t\end{array}$ \\
\hline PI-4 & $417,242,3 / 8.254$ & $\begin{array}{c}x=240+0.502 t \\
y=406+1.833 t \\
z=4+t\end{array}$ \\
\hline PI-5 & $404,258,5 / 9.105$ & $\begin{array}{c}x=256.2+0.999 t \\
y=405-0.01 t \\
z=8.5+t\end{array}$ \\
\hline PI-6 & $426,213,7 / 9,209$ & $\begin{array}{c}\mathrm{x}=218.3+0.012 \mathrm{t} \\
\mathrm{y}=394.2+1.261 \mathrm{t} \\
\mathrm{z}=17.05+\mathrm{t}\end{array}$ \\
\hline PI-7 & $422,211,13 / 8.537$ & $\begin{array}{c}\mathrm{x}=216.7-0.037 \mathrm{t} \\
\mathrm{y}=388.1+1.296 \mathrm{t} \\
\mathrm{z}=18.1+\mathrm{t}\end{array}$ \\
\hline PI-8 & $415,183,8 / 11.188$ & $\begin{array}{c}\mathrm{x}=182.2+5.423 \mathrm{t} \\
\mathrm{y}=415-0.027 \mathrm{t} \\
\mathrm{z}=2.012+\mathrm{t}\end{array}$ \\
\hline
\end{tabular}

Table 3. shows parameters specific to the proximal part of the femur.

Table 3. Parameters of the proximal part of the femur.

\begin{tabular}{|c|c|c|c|c|}
\hline Image & $\begin{array}{l}\text { Position of the } \\
\text { center }(x, y, z) \\
\text { and femoral } \\
\text { head diameter } \\
\quad[\mathrm{mm}]\end{array}$ & $\begin{array}{l}\text { Femoral neck } \\
\text { axis equation }\end{array}$ & $\begin{array}{l}\text { Fem. } \\
\text { neck } \\
\text { angle } \\
{\left[{ }^{0}\right]}\end{array}$ & $\begin{array}{l}\text { Offset } \\
{[\mathrm{mm}]}\end{array}$ \\
\hline CI-1 & $\begin{array}{c}240.8,345.9,259.5 \\
/ \\
44.66\end{array}$ & $\begin{array}{c}\mathrm{x}=240.8+105 \mathrm{t} \\
\mathrm{y}=345.9-86.28 \mathrm{t} \\
\mathrm{z}=259.5+25.14 \mathrm{t}\end{array}$ & $114.2^{0}$ & 61.223 \\
\hline CI-2 & $\begin{array}{c}271.1,345.4,183.5 \\
/ \\
43,62\end{array}$ & $\begin{array}{c}\mathrm{x}=271.1+34.62 \mathrm{t} \\
\mathrm{y}=345.4-32.2 \mathrm{t} \\
\mathrm{z}=183.5-27.47 \mathrm{t}\end{array}$ & $126.21^{0}$ & 45.412 \\
\hline CI-3 & $\begin{array}{c}262.0,344.1,228.6 \\
/ \\
46,12 \\
\end{array}$ & $\begin{array}{c}x=256.2+44.26 \mathrm{t} \\
\mathrm{y}=324.2-26.2 \mathrm{t} \\
\mathrm{z}=209.1-22.41 \mathrm{t}\end{array}$ & $119.21^{0}$ & 42.48 \\
\hline
\end{tabular}


As can be seen from the tables, morphological parameters whose determination results in functional dependency or coordinates cannot be compared with values obtained by other methods, while scalar values can be compared with results of measurements obtained using Able Software Corp 3D Doctor v3.5. software. Table 4. presents comparative parameter values obtained using these two softwares.

Table 4. Comparative parameter values.

\begin{tabular}{|c|c|c|c|c|}
\hline \multicolumn{2}{|l|}{ Image } & CI-1 & CI-2 & CI-3 \\
\hline \multirow[t]{3}{*}{ 3D Doctor } & $\begin{array}{l}\text { Femoral head } \\
\text { diameter } \\
{[\mathrm{mm}]}\end{array}$ & 47.45 & 43.58 & 44.80 \\
\hline & $\begin{array}{l}\text { Femoral neck } \\
\text { angle }\left[{ }^{0}\right]\end{array}$ & 115.97 & 116.89 & 121.31 \\
\hline & Offset $\left[{ }^{0}\right]$ & 50.47 & 48.05 & 44.02 \\
\hline \multirow{3}{*}{$\begin{array}{l}\text { Percentage } \\
\text { difference } \\
\text { compared } \\
\text { to the } \\
\text { developed } \\
\text { software }\end{array}$} & $\begin{array}{l}\text { Femoral head } \\
\text { diameter [\%] }\end{array}$ & 4.51 & 1.37 & 2.86 \\
\hline & $\begin{array}{l}\text { Femoral neck } \\
\text { angle }[\%]\end{array}$ & 1.53 & 3.29 & 1.72 \\
\hline & Offset [\%] & 6.50 & 3.62 & 3.50 \\
\hline
\end{tabular}

\section{DISCUSSION / CONCLUSIONS}

Procedures for diagnostic image processing, the method for determining femoral morphological parameters as well as developed software, can all be independently evaluated. Evaluation criteria can be: accuracy, applicability in research and/or practical application, and possibilities for further development.

The applied technique of diagnostic image processing includes a set of methods that are used in most scientific research and commercial softwares for diagnostic image processing [7, 22].

Approach to segmentation of bone images included an analysis of a large number of methods, using both theoretical and real results of specific CT images.

During the analysis of contour based methods in image border areas of the pelvic region (as in the proximal part of the femur, where the medullary canal ends), the LoG method provided the best results. The results obtained by using the LoG approach had the least unwanted pixels in the contour, which is observed in other studies [23]. Application of region based methods, mainly active contour methods, revealed a problem in previous analyses with the positioning of the initial mask and contour forming of the femur head (which is a very small distance away from the pelvis), so it is common that a formed contour includes a part of the pelvic region. In many cases, level set method application resulted in an inadequate formation of the bone contour section in the proximal region of the medullary canal end. This significantly complicates the determination of geometrical characteristics of the femoral head. In many cases, level set method application resulted in an inadequate formation of the bone contour section in the proximal region of the medullary canal end. Because of this, and based on previously obtained results, the LoG approach of image segmentation is implemented into software at this stage of its development.
Automated processing of metadata from pre-processed images, image correction according to the reconstruction of bone tissue and automatic determination of morphological characteristics of the femur, enables the femur reconstruction process and data processing to be implemented with speeds that correspond well with commercial software for this purpose $(20-30 \mathrm{~s})^{1}$ [24]. Factor that is somewhat slowing down image processing in the current stage of software development presents the need for manual definition of areas of importance to improve the bone tissue separation on each image. Therefore, in case of complex images (with more than 300 images in the recording) this phase requires up to $25-30 \mathrm{~min}$. of preparation activities. Further software development should include reduction of manual intervention and thus reduce the analysis time.

Femoral reconstruction, which is implemented using the developed software, is presenting the generally accepted method that is based on a series of $2 \mathrm{D}$ contours. Results in form of point clouds enable forming of files that can be imported into commercial CAD / CAE / CAM software and hence realization of endoprosthesis design. In addition, resulting contours can be approximated by polynomial curved lines and additional engineering parameters can be determined, which include the cement layer thickness (in cement prosthesis) and mathematical patterns (curved line) of femoral axis propagation. These activities present a basis for further research, as it is necessary to take the influence of distance between images in the DICOM file into account.

The process of morphological parameter determination is based on algebraic mathematical methods, applied on a number of points that describe the femur. Applicability of the procedure depends on the accuracy in determination of certain parameters, as well as errors that arise by applying methods for determination of shape from a set of points. Table 4 presents an overview of the results obtained by the developed software, compared to the measurement results acquired from the 3D Doctor software. Critical analysis of these results has to take into account that subjective error often occurs by using commercial software, since measurements are carried out manually. Its extent is comparable to the size of errors of the presented method. In addition, parameter accuracy can also present an issue: in determination of femoral head center and diameter, where the largest set of points, including unwanted pixels, is utilized; in defining anatomical axes of the femur, circles inscribed in the medullary canal and the definition of the sphere which describes the femoral head. Table 5 presents results of error analysis which occur due to the influence of unwanted pixels on femoral head parameter accuracy, and also includes average error deviation of defined femoral axis from the position of the centers of circles inscribed in the medullary canal.

From the error analysis results shown in Table 5., as well as comparative analysis of parameter measurements using

\footnotetext{
${ }^{1}$ Analyses were carried out on a computer with the following characteristics: CPU: i5 at $2.7 \mathrm{GHz}$, with $6 \mathrm{~GB}$ of RAM; and a 64 bit version of the Matlab 2010 software installed on it.
} 
commercial and developed software, it can be concluded that results are significant for endoprostheses design requirements. This is particularly evident if the precision of prosthesis surgery is taken into account. Incapability to determine the anteversion angle (the angle between femoral neck axis and coronal plane) can be considered as a drawback of the developed method. This is caused by the inability to determine the exact position of the coronal plane without reconstruction of the entire femur and pelvic region. Although method tests were carried out in order to indirectly calculate the femur position relative to the pelvis, this parameter was not determined at this stage of the research.

Table 5. Error analysis results.

\begin{tabular}{|l|l|l|l|}
\hline Image & CI-1 & CI-2 & CI-3 \\
\hline R spheres with & $44.662 /$ & $43.624 /$ & $46.121 /$ \\
/without unwanted \\
$\begin{array}{l}\text { points } \\
\text { [mm] }\end{array}$ & 44.664 & 43.630 & 46.125 \\
\hline Influence [\%] & 0.004 & 0.014 & 0.009 \\
\hline $\begin{array}{l}\text { Mean femoral axis } \\
\text { deviation from the } \\
\text { position of circles } \\
\text { centers inscribed in } \\
\text { the medullary } \\
\text { canal [mm] }\end{array}$ & 0.0043 & 0.0406 & 0.0341 \\
\hline
\end{tabular}

The developed software, as a tool that combines image processing methods and determination of femoral parameters, is based on object-oriented principles. This ensures its further partial development and analysis can be easily done. Results are exported in an ASCII file that contains reconstruction coordinates for CAD software, and in a text file containing all the morphological parameters determined by femoral analysis. The apparent disadvantages include use of manual point choice method (Region of Interest), which is used for selection of lesser trochanter and femoral head points, in order to avoid accidental selection of unwanted points that are occurring during the image processing. This significantly slows down the process of bone parameter determination.

The results obtained using the software for 12 diagnostic images confirm the hypothesis that geometrical parameters that are necessary for the design of total hip endoprosthesis can be successfully determined by using adequate image processing and analysis methods. From an engineering point of view, obtained results are acceptably accurate. Most importantly, the accuracy of obtained results does not depend on the image processing method or the morphological parameters determination method; it only depends on the recording process and parameters that are used during the recording phase. Analysis results are obtained from CT images, which is a drawback of the verification process. This is due to the insufficient number of MRI images created with different recording protocols. Attained results for several MRI images are encouraging, but insufficient for software evaluation.

Methods of image processing, determination of femoral geometrical parameters and created software, all present the first phase of research that aims to explore the possibilities for automatization of endoprosthesis design process in accordance with patient measures. This will enable significantly shorter time needed for development of custom endoprostheses and reduce the associated costs.

\section{ACKNOWLEDGMENT}

This work was supported by the Ministry of Education, Science and Technological Development of the Republic of Serbia, project No. TR 35025

\section{REFERENCES}

[1] Pawlikowski, M., Skalski, K., Haraburda, M. (2003). Process of hip joint prosthesis design including bone remodeling phenomenon. Computers \& Structures, 81 (8-11), 887-893.

[2] Ramos, A., Completo, A., Relvas, C., Simões, J.A. (2012). Design process of a novel cemented hip femoral stem concept. Materials \& Design, 33, 313321.

[3] Garellick, G., Karrbolm, J., Rogmark, C., Herberts, P. (2011). Annual Report 2010. Swedish Hip Arthroplasty Register.

[4] International Organisation for Standardization. (2008). Implants for surgery - Partial and total hip joint prostheses - Part 1: Classification and designation of dimensions. ISO 7206-1.

[5] Abadie, P., Lebel, B., Pineau, V., Burdin, G., Vielpeau, C. (2010). Cemented total Hip stem design influence on adaptative cortical thickness and femoral morphology. Orthopaedics \& Traumatology: Surgery \& Research, 96 (2), 104-110.

[6] Tabakovic, S., Zeljkovic, M., Zivkovic, A. (2013). General parametric model of the body of the total hip endoprosthesis. Acta Polytechnica Hungarica, 13.

[7] Yuanzhi, C., Shengjun, Z., Yadong, W., Changyong, G., Jing, B., Shinichi, T. (2013). Automatic segmentation technique for acetabulum and femoral head in CT images. Pattern Recognition, 46 (11), 2969-2984.

[8] Galibarov, P.E., Prendergast, P.J., Lennon, A.B. (2010). A method to reconstruct patient-specific proximal femur surface models from planar preoperative radiographs. Medical Engineering \& Physics, 32 (10), 1180-1188.

[9] Otomaru, I., Nakamoto, M., Kagiyama, Y., Takao, M., Sugano, N., Tomiyama, N., Tada, Y., Sato, Y. (2012). Automated preoperative planning of femoral stem in total hip arthroplasty from 3D CT data: Atlas-based approach and comparative study. Medical Image Analysis, 16 (2), 415-426.

[10] Rawal, B.R., Ribeiro, R., Malhotra, R., Bhatnagar, N. (2012). Design and manufacturing of femoral stems for the Indian population. Journal of Manufacturing Processes, 14 (3), 216-223.

[11] Yongtae, J., Kuiwoon, C. (2010). Design of patientspecific hip implants based on the 3D geometry of the human femur. Advances in Engineering Software, 41 (4), 537-547. 
[12] Ruben, R., Fernandes, P., Folgado, J. (2012). On the optimal shape of hip implants. Journal of biomechanics, 45 (2), 239-246.

[13] Kayabasi, O., Ekici, B. (2011). The effects of static, dynamic and fatigue behaviour on three-dimensional shape optimization of Kayabaşi_Ekici type hip prosthesis by finite element method and probabilistic approach. Journal of Biomechanics, 44, 6.

[14] Sridhar, I., Adie, P.P., Ghista, D.N. (2010). Optimal design of customised hip prosthesis using fiber reinforced polymer composites. Materials \& Design, 31 (6), 2767-2775.

[15] International Organisation for Standardization. (2011). Health informatics -- Digital imaging and communication in medicine (DICOM) including workflow and data management. ISO 12052.

[16] Li, X. (2010). Semi-automatic segmentation of normal female pelvic floor structures from magnetic resonance images. Unpublished doctoral dissertation. Cleveland State University, USA.

[17] Kale, E.H., Mumcuoglu, E.U., Hamcan, S. (2012). Automatic segmentation of human facial tissue by MRI-CT fusion: A feasibility study. Computer Methods and Programs in Biomedicine, 108 (3), 11061120 .

[18] Gamage, P., Xie, S.Q., Delmas, P., Xu, W.L. (2011). Diagnostic radiograph based $3 \mathrm{D}$ bone reconstruction framework: Application to the femur. Computerized Medical Imaging and Graphics, 35 (6), 427-437.
[19] Wang, J., Ye, M., Liu, Z., Wang, C. (2009). Precision of cortical bone reconstruction based on 3D CT scans. Computerized Medical Imaging and Graphics, 33 (3), 235-241.

[20] Hauser, J.R. (2009). Numerical Methods for Nonlinear Engineering Models. Springer.

[21] Mahaisavariya, B., Sitthiseripratip, K., Tongdee, T., Bohez, E., Sloten, J.V., Oris, P. (2002). Morphological study of the proximal femur: A new method of geometrical assessment using 3-dimensional reverse engineering. Medical Engineering \& Physics, 24 (9), 617-622.

[22] Ababneh, S., Prescott, J., Gurcan, M. (2011). Automatic graph-cut based segmentation of bones from knee magnetic resonance images for osteoarthritis research. Medical Image Analysis, 15 (4), 438-448.

[23] Muhit, A.A., Pickering, M.R., Scarvell, J.M., Ward, T., Smith, P.N. (2013). Image-assisted non-invasive and dynamic biomechanical analysis of human joints. Physics in Medicine and Biology, 58, (13), 4679-4702.

[24] Van Cauter, S., De Beule, M., Han Haver, A., Verdonk, P., Verhegghe, B. (2012) Automated extraction of the femoral anatomical axis for determining the intramedullary rod parameters in total knee arthroplasty. International Journal for Numerical Methods in Biomedical Engineering, 28, 158-169.

Received January 03, 2014. Accepted September 30, 2014. 